CARNETS OE Carnets de géographes

GÉOGRAPHES.

7| 2014

Les espaces de l'entre-deux

\title{
Le cortiço : logement populaire de l'entre-deux
}

\section{Octavie Paris}

\section{OpenEdition}

Journals

Édition électronique

URL : http://journals.openedition.org/cdg/514

DOI : $10.4000 /$ cdg. 514

ISSN : 2107-7266

\section{Éditeur}

UMR 245 - CESSMA

\section{Référence électronique}

Octavie Paris, «Le cortiço : logement populaire de l'entre-deux », Carnets de géographes [En ligne],

7 | 2014, mis en ligne le 01 décembre 2014, consulté le 19 avril 2019. URL : http:// journals.openedition.org/cdg/514; DOI : 10.4000/cdg.514

\section{c) $(1) \Theta$}

La revue Carnets de géographes est mise à disposition selon les termes de la Licence Creative

Commons Attribution - Pas d'Utilisation Commerciale - Pas de Modification 4.0 International. 


\title{
LE CORTIÇO : LOGEMENT POPULAIRE DE L'ENTRE-DEUX
}

\author{
OCTAVIE PARIS \\ Université Lyon 3 \\ Géographie \\ octavie.paris@ens-lyon.fr
}

\section{Résumé :}

Le terme cortiço désigne historiquement un logement populaire, collectif, locatif et multifamilial du centre des grandes villes brésiliennes. Bien que nombre de cortiços aient été détruits sous l'effet de réformes urbaines successives dans une partie de ces espaces (comme à Rio de Janeiro), ces logements demeurent des figures importantes de l'habitat des centres-ville. Cet article propose une comparaison de I'habitat en cortiço à Sao Paulo et Recife à partir d'un double regard à la fois temporel, porté sur les trajectoires de vie de ses habitants, et spatial, considérant leur accès à la ville. Entre-deux terminologique, le cortiço est difficile à appréhender tant par les politiques publiques que par le chercheur lui-même. Sur un plan plus concret, le cortiço comme élément bâti, se présente également comme un espace de l'entre-deux dans le paysage urbain dans lequel il s'insère, à différentes échelles (ville, quartier, rue). L'étude du cortiço en regard des trajectoires de vie de ses habitants et de leurs micro-appropriations de l'espace éclaire davantage encore cette notion d'entredeux, le rapport à l'espace domestique étant ici appréhendé comme un révélateur du rapport à la ville. Ainsi, loin de toute approche manichéenne, la situation d'entre-deux qui peut caractériser la réalité urbaine populaire que constitue le cortiço n'est pas appréhendée négativement par ses habitants. Elle leur apporte en effet aussi de nombreux avantages matériels et immatériels. Le cortiço éclaire ainsi le sens concret, spatial et donc physique de l'entre-deux, autant que son sens plus abstrait, plus psychologique et social.

\section{Abstract :}

The word cortiço is traditionnally linked to a popular, collective, rental and multifamily housing in the center of the big Brasilian cities. Despite some of them have been destroyed by successive urban reforms in part of these spaces, these housings keep on being major images of the city centers. This article deals with a comparison between the cortiço housing through a double point of view, one temporal on the inhabitants' life-courses, and one spatial, considering their access to the city. In-between, the cortiço is hard to grasp for the public policies as for the researcher. In a more concrete way, the cortiço as a building, appears also as an in-between space in the urban landscape in which he exists in various scales (city, neighborhood, street). Studying the cortiço throught the inhabitants' life courses and their micro-approriations enlights this notion of in-between. The access to the city framework permits the analyse of the cortiço in this text throught the micro-appropriations 
and the link to the domestic space revealing the link to the city. So, away from any manichean approach, the in-between does not appear exclusively negative in the apprehension of this urban popular reality that the cortiço is but much more like a decisive caracteristic for the inhabitants who find a lot of advantages in it. This in-between idea is taken in a concrete sense, spatial and physic, but also in a more abstract one, as a psychological and social reality.

\section{Introduction}

Lorsque l'on aborde le thème de l'habitat populaire dans les grandes villes brésiliennes, il est courant de penser à la favela, paysage urbain mondialement connu. La prédominance de I'habitat en favela dans l'imaginaire commun ou dans les politiques publiques qui concernent l'habitat populaire au Brésil est certainement due, entre autres facteurs, à son importante médiatisation et à sa portée sur les écrans de cinéma ${ }^{1}$. Ces représentations s'opèrent au détriment d'autres types d'habitats populaires tout aussi caractéristiques de la réalité urbaine du géant d'Amérique du Sud. Parmi eux, le cortiço, objet de cet article.

Le terme cortiço désigne un logement populaire, collectif, locatif et multifamilial des centres anciens des villes brésiliennes. En français, le terme équivalent est souvent «taudis ", mais cette traduction apparaît trop simpliste, car le mot " cortiço » file la métaphore de la ruche $\mathrm{d}^{\prime}$ abeille organisée en cortiça ${ }^{2}$. C'est donc l'image de bourdonnement et de surpopulation

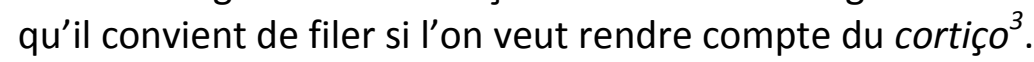

Les cortiços occupent d'anciennes maisons coloniales, situées en centre-ville, mais invisibles aux yeux des citadins qui, soit ne soupçonnent pas que ce logement aux apparences unifamiliales puisse héberger de très nombreuses familles, soit l'assimilent à un squat. Or, contrairement à ce dernier, qui indique le fait d'habiter "sans contrat un local vacant " [Bouillon, $2010:$ 14], le cortiço est fondé sur un système de location et de sous-location de quartos ${ }^{4}$ avec un contrat, tacite et oral mais néanmoins existant, selon lequel l'habitant paye un loyer directement au propriétaire ou au(x) responsable $(s)^{5}$. Dans les trajectoires de vie, le cortiço apparaît comme une solution de logement souvent temporaire, mais certains s'y établissent de manière plus définitive. L'ensemble de ces caractéristiques, entre formel et informel, visible et invisible, temporaire et pérenne, font du cortiço un objet difficile à cerner. Ce carnet de recherche nous donne l'occasion d'explorer cet objet spatial hybride au prisme de la notion d'entre-deux et d'interroger la pertinence du concept pour mieux approcher cette forme d'habitat populaire et ce mode d'habiter.

A ce stade, nous considérons l'entre-deux comme un espace intermédiaire, une sorte de zone tampon entre deux types d'espaces qui ne cesse pour autant d'en faire partie. Dans ce

\footnotetext{
${ }^{1}$ Nombreux sont les films et séries de portée internationale ayant pour scène les favelas : Cidade de Deus (Meirelles F., Lund K., 2002) , Tropa de Elite (Padilha J., 2007) pour n'en citer que deux.

2 Liège.

${ }^{3}$ Il apparaît donc plus adéquat de ne pas adopter cette traduction, en gardant le terme cortiço dans la langue source.

${ }^{4}$ Littéralement "chambres" en français, mais il s'agit là d'une pièce unique multifonctionnelle où vivent plusieurs personnes d'une même famille, n'ayant pas toujours de fenêtre donnant sur l'extérieur et la rue.

${ }^{5}$ Précisons toutefois qu'historiquement, ce type de logement n'avait rien d'informel. Cette considération dépend également de la ville étudiée et la situation de São Paulo n'est pas comparable à celle de Rio de Janeiro qui a connu de nombreuses opérations urbaines de modernisation dans le centre-ville [Vaz, 1994: 90]. Le propriétaire confie dans certains cas, la collecte des loyers à une tierce personne.
} 
cadre assez large, nous proposons plus précisément de rapprocher l'entre-deux de la figure du "sas", entendu comme "une enceinte ou passage clos, muni de deux portes ou systèmes de fermeture dont on ne peut ouvrir l'un que si l'autre est fermé et qui permet de passer ou de faire passer d'un milieu à un autre en maintenant ceux-ci isolés l'un de l'autre " (Larousse). Malgré son invisibilité ou sa faible visibilité dans le paysage urbain, comment le cortiço constitue-t-il à la fois un sas spatio-temporel dans la ville pour les populations qui I'habitent et un lieu et un moment d'ancrage dans les charnières des trajectoires de vie [Chabrol, $2011: 71]$, qui permet à ses habitants d'accéder à la ville et à la citadinité [Gervais Lambony, 2001] ? L'invisibilité [Voirol, 2005: 10] ne serait pas alors une caractéristique négative dans le cas des cortiços, puisqu'elle se conjuguerait et participerait à l'avantage fondé sur la façade et le camouflage et la localisation en centre-ville.

Ces questionnements s'appuient sur deux expériences de recherche sur l'objet cortiço ${ }^{6}$ dans deux grandes métropoles brésiliennes présentant des réalités urbaines bien distinctes: Recife et São Paulo (Figure 1). Recife, capitale de l'Etat du Pernambouc située dans le Nordeste, une région considérée et stigmatisée comme l'une des plus pauvres du pays, est connue pour l'importance et la diversité de l'habitat populaire dans son tissu urbain central. São Paulo, mégalopole et capitale économique du pays, est située, elle, dans la région SudEst du Brésil, l'une des plus développées du pays, et attire ainsi nombre de populations migrantes nationales à la recherche d'un avenir meilleur ou différent de leur situation d'origine. Les solutions de logement populaire y sont donc particulièrement diversifiées mais celle du logement en cortiço reste importante : de façon significative, la seule définition officielle du cortiço existant à ce jour est une loi municipale de São Paulo, appelée Lei Moura $^{7}$. Il apparaissait donc intéressant d'étudier la place du cortiço dans le large panel des logements populaires de Recife et de São Paulo.

Nous présenterons d'abord deux façons de repérer et d'aborder le cortiço sur le plan méthodologique, ce qui participe pleinement à faire de cet espace un entre-deux pour la recherche. Puis nous proposerons deux analyses du rapport à la ville de ses habitants pour interroger la fonction de "sas" du cortiço: en observant d'une part leurs logiques $d^{\prime}$ 'installation, d'appropriation et d'ancrage, et d'autre part, leurs logiques mobilitaires intraurbaines.

\footnotetext{
${ }^{6}$ Mémoires de Master 1 \& 2 STADE (ENS de Lyon) de l'auteure : "Géographie de l'invisibilité urbaine : les cortiços de Bela Vista à São Paulo " (2012); "Habiter en cortiço à Recife (Brésil) : Pratiques et logiques citadines de résidents pauvres en centre-ville» (2013)

${ }^{7}$ Loi Moura $n^{\circ} 10.928$ datant de 8 octobre 1991

" Art. $1^{\circ}$ - Le cortiço est défini comme une unité utilisée comme habitat collectif multifamilial présentant, totalement ou partiellement, les caractéristiques suivantes:

a) Constituée d'une ou de plusieurs édifications construites sur un sol urbain ;

b) Subdivisée en plusieurs pièces louées, sous-louées ou cédées à n'importe quel titre ;

c) Diverses fonctions exercées par une même pièce ;

d) Accès et usage commun des espaces non construits et des installations sanitaires ;

e) Circulation et infrastructure, en général précaires

f) Surpopulation d'habitants »
} 
Figure 1 : Localisation des Etats de São Paulo et du Pernambouc (São Paulo \& Recife)

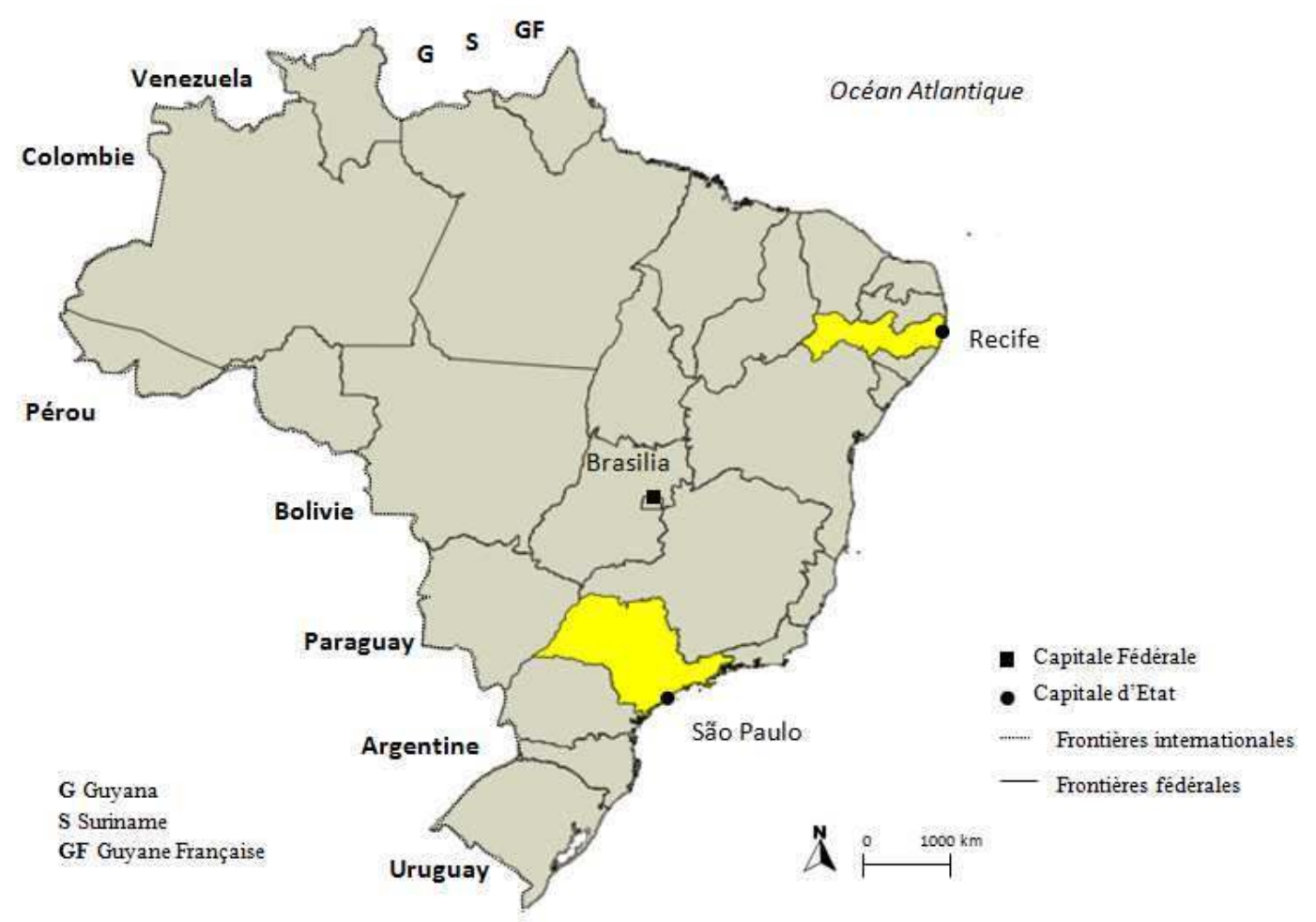

CPARIS O., Août 2013

Réalisation : Auteure, Août 2013

\section{Approcher le cortiço, invisible des statistiques et des normes brésiliennes sur l'habitat populaire}

Le cortiço n'appartient à aucune catégorie statistique précise, ce qui rend son appréhension des plus difficiles pour le chercheur. Alors qu'une catégorie spéciale est dédiée à l'habitat en favela dans les recensements de l'IBGE ${ }^{8}$, une recherche minutieuse par le biais de nombreux critères est nécessaire avant d'accéder aux données sur le logement en cortiço ${ }^{9}$. Officiellement, il est souvent qualifié de Habitação Coletiva Precária de Aluguel [Habitat Collectif Précaire Locatif] ou HCPA, mais ce sigle n'empêche pas de nombreuses confusions terminologiques, puisque l'expression englobe d'autres formes d'habitats populaires.

Le chercheur doit donc jongler entre ces dernières pour proposer finalement une définition en creux du cortiço, adaptée à chaque environnement urbain. Les cortiços n'accueillent pas la même population que les pensões ${ }^{10}$ ou les palafitas (cabanons sur pilotis au-dessus d'un

\footnotetext{
${ }^{8}$ Instituto Brasileiro de Geografia e Estadística - Institut Brésilien de Géographie et de Statistique

${ }^{9}$ Le cortiço est comptabilisé dans la même catégorie que d'autres logements populaires qui, bien que partageant certaines caractéristiques, ne sont en rien similaires, notamment du point de vue de la modalité de logement (propriété, location, paiement de loyer ou non). La catégorie est référencée en « domicile permanent particulier, improvisé, collectif » dans les divisions de l'IBGE.

${ }^{10}$ Traduit par "pensions". Bien que le mode d'habiter soit très proche de celui en cortiço, certaines caractéristiques de la population résidente diffèrent: la dimension multifamiliale n'est pas présente et la population est volontairement homogène, souvent il est spécifié "pension pour femmes sans enfants ", " pension pour hommes ", " pensions pour retraités » discrètement vers la porte d'entrée.
} 
cours d'eau ou d'une étendue d'eau, où vivent généralement des personnes seules et/ou des couples sans enfants). Ils ne présentent pas non plus les mêmes modalités de logement que les mocambos (cabanes lacustres -originellement de paille- où se cachaient les esclaves d'origine africaine [Freyre, 1936]) ou les invasões ("invasion " d'un bâtiment vide ou inoccupé sans paiement de loyer). Enfin, à l'inverse des différents types d'habitats cités et de la favela, qui partagent tous un certain degré de visibilité dans le panorama de la ville et disposent donc d'une place centrale dans les politiques publiques pour le logement, le cortiço ne se repère pas facilement dans le paysage urbain quotidien. La favela, par exemple, se démarque facilement par son étendue spatiale [Vaz, 1994], de même que les palafitas, qui surplombent les fleuves (Erreur ! Source du renvoi introuvable.), sont reconnaissables depuis n'importe quel pont de la ville de Recife.

\section{Figure 2 : Palafitas sur les rives du fleuve Capibaribe, à Recife}

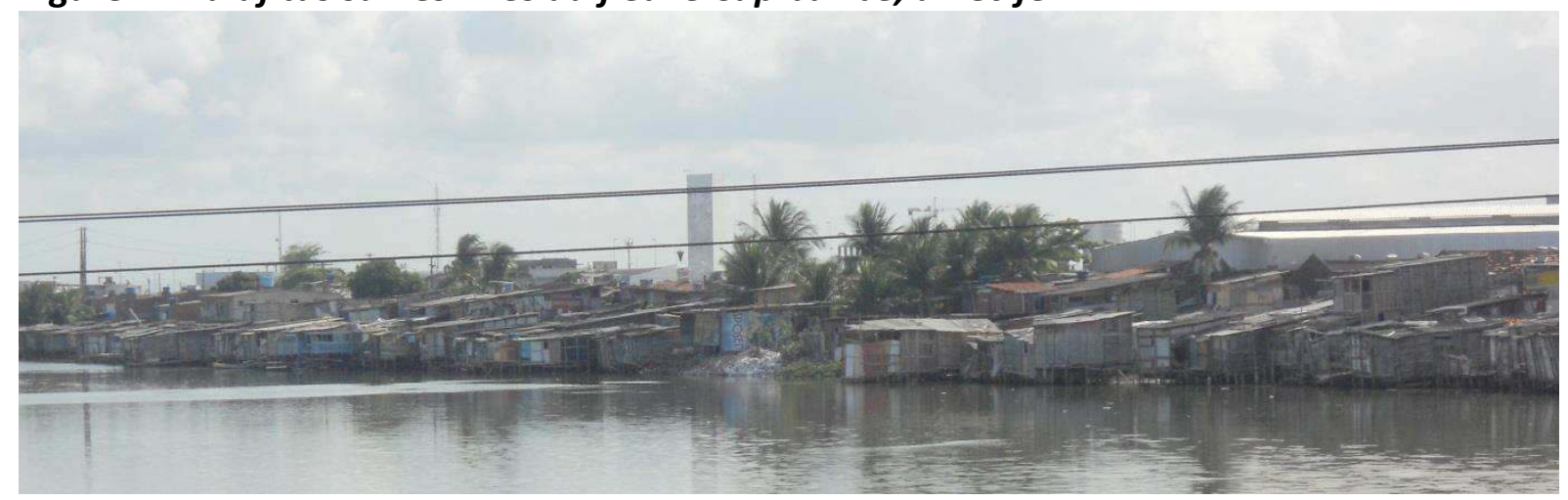

Cliché : Auteure, Recife, 22 Février 2013

Devant un tel cadre statistique, prétendre au quantitatif et à l'exhaustif sur le thème et l'espace choisis paraissait ambitieux. En conséquence a été privilégiée une approche par l'échelle micro, offrant une richesse et une finesse qualitatives. Deux terrains de quatre mois chacun ont permis d'étudier des cortiços de deux quartiers centraux et anciens, Bela Vista à São Paulo et Boa Vista à Recife. Dans le premier cas, dix cortiços ont été appréhendés pour un total de 33 habitants interrogés. Dans le second, nous avons recherché l'exhaustivité des habitants par cortiço: l'étude s'est donc concentrée sur seulement deux cortiços, en assurant la rencontre avec la quasi-totalité des habitants et la réalisation de questionnaires avec un habitant par quarto, soit 16 au tota $\left.\right|^{11}$. A part ces questionnaires réalisés auprès des habitants, des entretiens ont été proposés à des professionnels gravitant autour des cortiços, tels les institutions municipales et étatiques, les ONG, les services publics de santé et de police, ou encore les promoteurs immobiliers. En outre, une enquête de rue a été menée dans le quartier de Bela Vista afin d'avoir une idée des représentations que les citadins se faisaient du cortiço et de voir s'ils pouvaient en donner une définition ou une description. Le travail de recherche sur le terrain a donc consisté à jongler avec cette position d'entre-deux dans lequel se trouve l'habitat en cortiço : devant l'impossibilité de se limiter aux recensements officiels qui n'intègrent aucune catégorie spécifique et uniformisée pour l'habitat en cortiço, tenter néanmoins d'appréhender autrement cet habitat peu connu des grandes villes brésiliennes.

\footnotetext{
${ }^{11}$ Les refus et absences prolongées de certains habitants n'ont pas permis d'atteindre l'exhaustivité totale.
} 


\section{L'habitat populaire invisible ? Le cortiço dans le paysage urbain des villes brésiliennes}

Avant même de proposer cette démarche qualitative, nous avons dû repérer le cortiço dans le paysage urbain. Le paysage ne se présente pas seulement comme un tableau statique et immuable, mais aussi comme un moyen d'analyse en géographie [Rimbert, 1973], qui reflète "le côté sensible de la relation d'une société à l'espace " [Berque, 1995]. Par conséquent, analyser le cortiço non comme un élément isolé, mais comme un élément du paysage urbain auquel il appartient, aide à définir son régime de visibilité, entendu comme "l'advenue au visible, cette présentation aux regards [qui permet] que l'existence [des objets] au sein de la société se cristallise » [Lévy, Lussault, 2003 : 997]. Ce régime de logement dépend non seulement de caractéristiques physiques du bâti, du statut d'occupation, de sa localisation, mais aussi des modes de vie des populations qui y vivent. Ainsi, considérer la morphologie et la typologie générale de la ville dans lequel s'insère le cortiço en regard de ses propres caractéristiques morphologiques permet de mieux comprendre sa place dans la ville brésilienne.

A l'échelle des villes de Sao Paulo et Recife, le cortiço se distingue difficilement. Le centre de la capitale pauliste est particulièrement verticalisé : sa skyline est parmi les plus hautes du monde [Erreur! Source du renvoi introuvable.], si bien que la partie basse du quartier de Bela Vista s'y trouve fondue visuellement dès que le regard prend de la hauteur. Le centre de Recife, lui, n'a pas connu un processus d'urbanisation aussi vigoureux et reste caractérisé par des bâtiments peu élevés. Est-ce pour autant que le cortiço y est davantage donné à la vue ? Si à São Paulo, le cortiço est perdu dans la forêt de gratte-ciels [Monbeig, 1953 : 289], il ne semble pas plus se démarquer dans le panorama général de la ville de Recife. Pour adopter un point de vue " par le haut » comme depuis la tour Banespa de São Paulo, on peut monter aux derniers étages de l'immeuble de la Mairie de Recife : le cortiço apparaît fondu dans " l'habitat bas " de la ville, d'un ou deux étages tout au plus. Dans les deux cas, la faible visibilité de cette solution de logement populaire s'explique par son caractère ponctuel et relativement dispersé. A l'inverse des favelas, le cortiço ne s'étale pas sur de grandes portions d'espace. Inséré dans la ville formelle, il ne bénéficie pas de la même attention que ces baraquements qui se donnent facilement à la vue et donc sur lesquels toute intervention des pouvoirs publics sera distinguable aisément, servant ainsi un argumentaire des politiques d'intervention sur le logement. 
Figure 3 : Skyline de São Paulo depuis la tour BANESPA 
ne s'agit pas pour eux de considérations d'ordre architectural, mais d'une façon de décrire la division interne, le manque d'espace face au surpeuplement du logement et des quartos. Ces éléments de morphologie, observés par nos soins ou relevés par nos interlocuteurs, participent à donner au cortiço un faible régime de visibilité. Ils obstruent donc d'autant plus une prise de conscience de la situation et des besoins de ce type de logement populaire de la part des autorités publiques. Et si à São Paulo, un programme a été mis en place, dit "Programme d'Action dans les Cortiços" (PAC) en association avec la compagnie responsable de l'habitat social dans l'Etat de São Paulo (CDHU), il n'est plus en fonctionnement depuis 2010. Au-delà, ces caractéristiques physiques du bâti, et notamment la profondeur du cortiço, participent à le qualifier d'interstice, le long couloir (Figure 4) semblant séparer une façade d'un logement unifamilial de l'intérieur d'un logement collectif, surpeuplé.

Figure 4 : L'étroitesse du bâti des cortiços : la façade (une simple porte) et l'intérieur
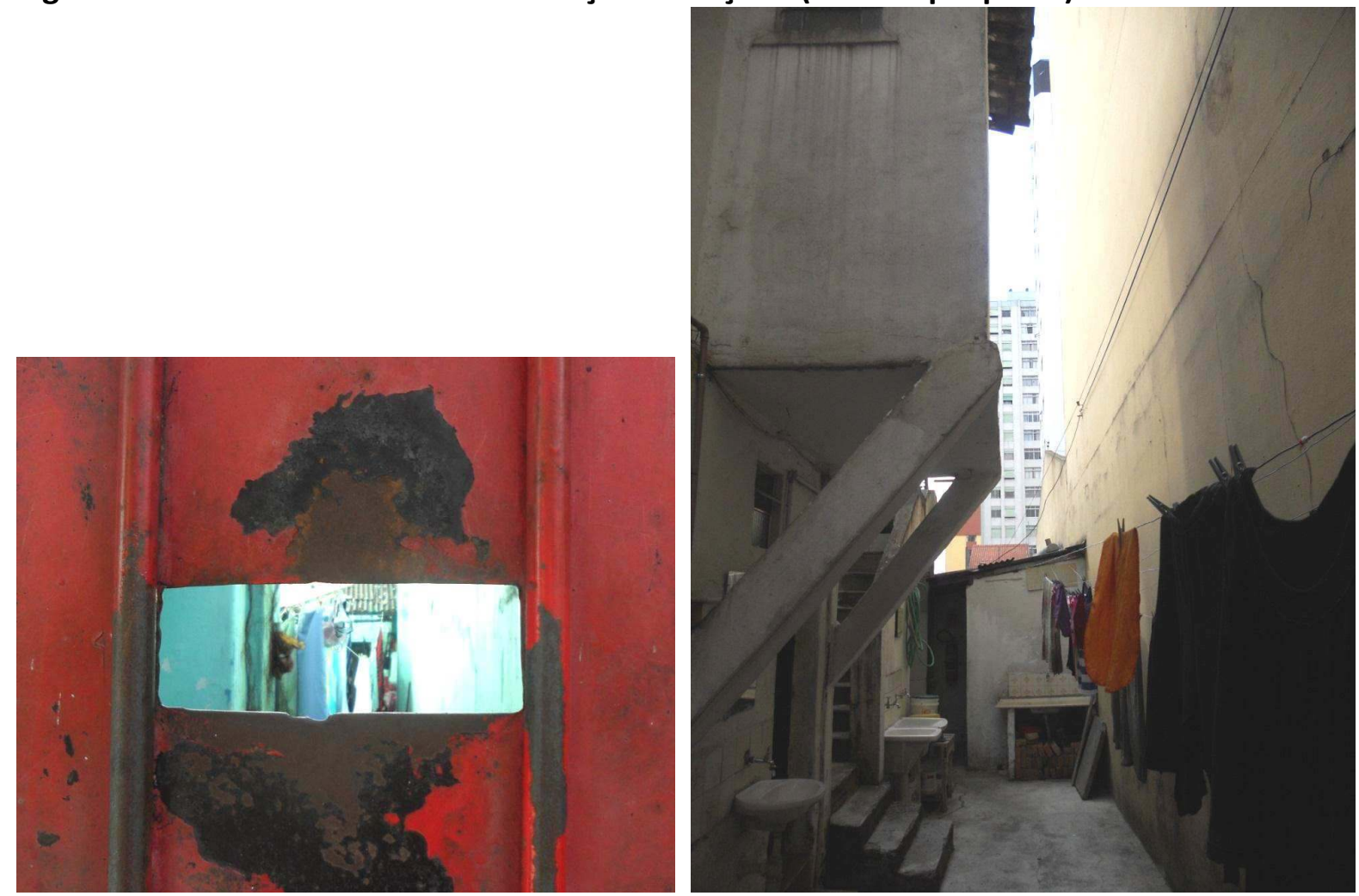

Clichés : Auteure, Bela Vista - São Paulo - Avril 2012

Cependant, cette notion d'interstice n'est pas à prendre dans le sens d'un non-construit, ni de " délaissés urbains, de friches et de terrains vagues, [où le vague signifierait] l'indéfini, l'indéterminé, l'ouverture dans la ville. " [Petcou et alii, 2005]. II s'agit plutôt d'espaces à fortes potentialités pour les populations habitantes du fait d'une certaine « résistance à I'homogénéisation et à l'appropriation définitive " [Petcou et alii, 2005]. Cette indéfinition, invite à dépasser alors les considérations architecturales du cortiço et à interroger la relation entre ce type d'habitat, ses habitants et le reste de la ville : comment le mode d'habitat en cortiço favorise ou au contraire limite l'accès à la ville de ses habitants, notamment leur accessibilité aux services et aux aménités du système urbain dans lequel il s'inscrit ? L'étude plus précise des populations qui y vivent, de leurs trajectoires de vie et de leurs modes 
d'appropriation de l'espace intra et extra cortiço, offre de nouvelles perspectives pour présenter cet habitat.

\section{Ancrage dans le sas : le cortiço, une étape majeure des trajectoires de vie.}

Au-delà de l'observation du bâti, l'étude approfondie des cortiços de São Paulo et de Recife nous a permis d'interroger soixante habitants. II s'agit principalement de familles de quatre personnes en moyenne, certaines familles plus nombreuses portant à six personnes le nombre d'habitants dans l'espace réduit du quarto, qui constitue l'unité de logement. L'hétérogénéité de cette population s'illustre également par la présence de résidents célibataires, avec ou sans enfants, ainsi que par l'étendue des tranches d'âge : le plus jeune chef de famille de nos enquêtes est une jeune maman de 18 ans et l'habitant le plus âgé est un retraité de 89 ans. Dans la mesure où ils paient un loyer, on ne peut pas parler de populations pauvres ou très pauvres : la majorité d'entre eux travaille et répond plus aux caractéristiques de la précarité que de la pauvreté. Les méthodes déployées ont permis de dresser un portrait presque exhaustif des cortiços étudiés et avec le traitement des données recueillies dans l'ensemble des questionnaires faits aux habitants, il est possible de reconstruire certaines trajectoires de vie [Lévy-Vroelant, 2008] [Figure 5]. Comment le cortiço se révèle-t-il être un espace de passage, une étape transitoire et, en ce sens, un sas dans les trajectoires de vie des habitants ? Comment, paradoxalement, observe-t-on un ancrage des populations dans cette situation de transition ? Ces éléments nous invitent non seulement à travailler le caractère temporel / temporaire du sas, mais aussi à observer et interpréter les marques d'appropriation des espaces de l'entre-deux.

\section{Un sas migratoire, familial, professionnel, résidentiel}

Les caractéristiques socio-démographiques des habitants des cortiços étudiés mettent en évidence trois récurrences dans le choix de logement en cortiço: l'origine migrante des populations, la relation de ses occupants à l'emploi urbain, et le moment de vie où ils s'installent.

Les premiers cortiços ont émergé avec les vagues d'immigration d'Européens et d'Italiens notamment dans les capitales pauliste et carioca ${ }^{14}$ au moment du boom industriel à la fin du XIXème siècle.

« Une grande quantité d'immigrants arrivaient dans la ville pour occuper des espaces de travail dans les usines et les industries. Ces travailleurs, généralement rémunérés avec de bas salaires, n'avaient pas les ressources pour acquérir un logement ou pour payer un loyer en habitat unifamilial. Ainsi, pourtant interdits, les cortiços sont devenus l'option de logement la plus viable et la plus courante de cette partie de la population. ${ }^{15}$ [Lopez, França \& Prado Costa, 2010 : 19]

Aujourd'hui encore, dans de nombreux cas, les cortiços offrent une solution de logement à des populations migrantes. A São Paulo, la majorité des habitants des cortiços étudiés proviennent ainsi d'autres régions du pays. Néanmoins, ce n'est pas toujours le cas: à

\footnotetext{
${ }^{14}$ São Paulo et Rio de Janeiro

15 « Uma grande quantidade de imigrantes chegava à cidade para ocupar espaços de trabalho em fábricas e indústrias. Esses trabalhadores, geralmente remunerados com baixos salários, não tinham condições de adquirir um imóvel ou de pagar aluguéis nas habitações unifamiliares. Assim, de proibidos os cortiços se tornaram a opção de moradia mais viável e recorrente dessa parcela da população.
} 
Recife, la majorité des habitants sont originaires de la ville, parfois du quartier même, et seulement dans quelques cas de l'Etat du Pernambouc ou de la région nordestine.

Ces différences entre les contextes urbains étudiés nous a amenée à travailler sur d'autres critères que l'origine des populations. Initialement, le cortiço est une solution de logement pour les populations migrantes en provenance du Vieux-Continent ayant trouvé un emploi dans les industries du centre. Aujourd'hui, le centre des grandes villes brésiliennes concentre encore la plupart des emplois, notamment informels [Théry, 2004 ; Cavalcanti, 2008]. Aussi, contrairement aux idées reçues, le cortiço reste une solution de logement pour une population de travailleurs et non de personnes marginalisées, sans emplois. Sa localisation permet en effet une proximité au centre et au lieu de travail et, par conséquent, un net avantage distance / temps face aux difficultés de transport urbain. En ce sens, le cortiço apparaît comme un sas pour l'accès à l'emploi.

Enfin, le cortiço est souvent investi à des étapes charnières de la vie de ceux qui y habitent : l'indépendance du foyer familial, le premier enfant ou encore le passage à un autre statut social tel que celui de retraité. En témoignent les deux trajectoires de vie recueillies à Recife, celles de Fabiana, jeune maman de 19 ans installée dans le premier cortiço depuis deux ans, et d'Alfredo, retraité de 89 ans vivant dans le second cortiço depuis douze ans (Figure 5). Ainsi, pour la majorité des habitants (la situation d'Alfredo semble faire exception du fait de son âge), le cortiço constitue une solution de logement temporaire dans lequel s'installent des familles pour une durée limitée, dans l'attente d'un mieux (

Figure 6). Dans le prolongement de cette idée, précisons que la rotation entre les différents cortiços du même quartier est courante, ce qui corrobore aussi l'importance du choix du quartier plus que du cortiço en lui-même.

\section{Figure 5 : Portrait de vie de deux habitants de cortiços}

Alfredo, retraité de 89 ans, doyen du cortiço dans lequel il vit depuis douze ans, pose un regard passionné et profondément réfléchi sur son lieu de vie. Ancien agent administratif dans le secteur de l'industrie, ce gaúcho ${ }^{16}$ m'explique avoir choisi son lieu de vie et s'y plaire. Pour la somme de 190 Reais par mois, il vit dans la partie de la ville qui lui plait le plus, le centre. "De todas as cidades onde eu morei, sempre foi no centro", où il vit d'ailleurs depuis quarante ans. Il a depuis toujours ses habitudes dans le quartier où il peut se déplacer à pied, pour aller faire ses courses au marché de Boa Vista. L'église, il n'y va pas, l'octogénaire se déclare athée et contre toute religion. Ce qu'il aime, lui, c'est aller jusqu'au Parque 13 de Maio pour jouer aux échecs. S'il connaît très bien la ville, il quitte très peu son quartier, parfois il prend le bus pour rendre visite à sa famille d'adoption : celui qu'il considère comme son petit-fils vit à Mustardinha. Aux premières paroles, la vie dans le cortiço est des plus agréables, mais il en viendra très vite à me conseiller de façon subtile de ne pas parler à certains habitants qu'il juge sans éducation et sans savoir-vivre. La propriétaire? II a l'air de l'apprécier et d'entretenir de bonnes relations avec elle, car elle semble s'investir dans le bon fonctionnement de son bien immobilier en étant présente.

\footnotetext{
${ }^{16}$ Surnom donné aux brésiliens vivant dans la région Sud du pays. Né à Pelotas dans l’Etat du Rio Grande do Sul.
} 
Fabiana habite le premier quarto, à l'entrée du cortiço. Avec son fils Miguel, 7 mois, dans les bras, elle m'explique qu'elle vit avec son mari depuis deux ans dans le seul quarto du cortiço à posséder des toilettes. C'est aussi l'un des seuls à jouir d'une lumière naturelle par la présence de la grande fenêtre donnant sur la rue. Sa mère et ses sœurs, auxquelles elle rend visite régulièrement, à pied, avec son fils habitent dans le centre également. Elle a mis ses études de droit entre parenthèse avec l'arrivée de Miguel à 19 ans, mais elle compte bien les reprendre. Fabiana est une jeune fille sociable, elle aime sortir avec ses amis et sa famille. Lorsqu'ils vont à la plage c'est à Pina qu'ils se rendent. Boa Viagem? Elle déteste! Ce qu'elle aime c'est le désordre et l'agitation. D'ailleurs, un ami fait une entrée fracassante dans le quarto à ce moment-là et se sert dans le réfrigérateur, une visite éclair le temps d'embrasser son amie et il repart comme il était venu. Le suit de peu la sœur cadette de Fabiana qui passait dans le quartier avec une amie. Fabiana aime son logement, le seul défaut c'est qu'il soit un peu petit pour trois personnes, elle surnomme son logement "ovo"17 ou encore "burraco"18. Elle utilise d'ailleurs fréquemment le pas de porte comme une extension de son logement.

Rédaction : Auteure, Avril 2013

Figure 6 : Ancienneté des habitants dans le cortiço : du temporaire qui dure.

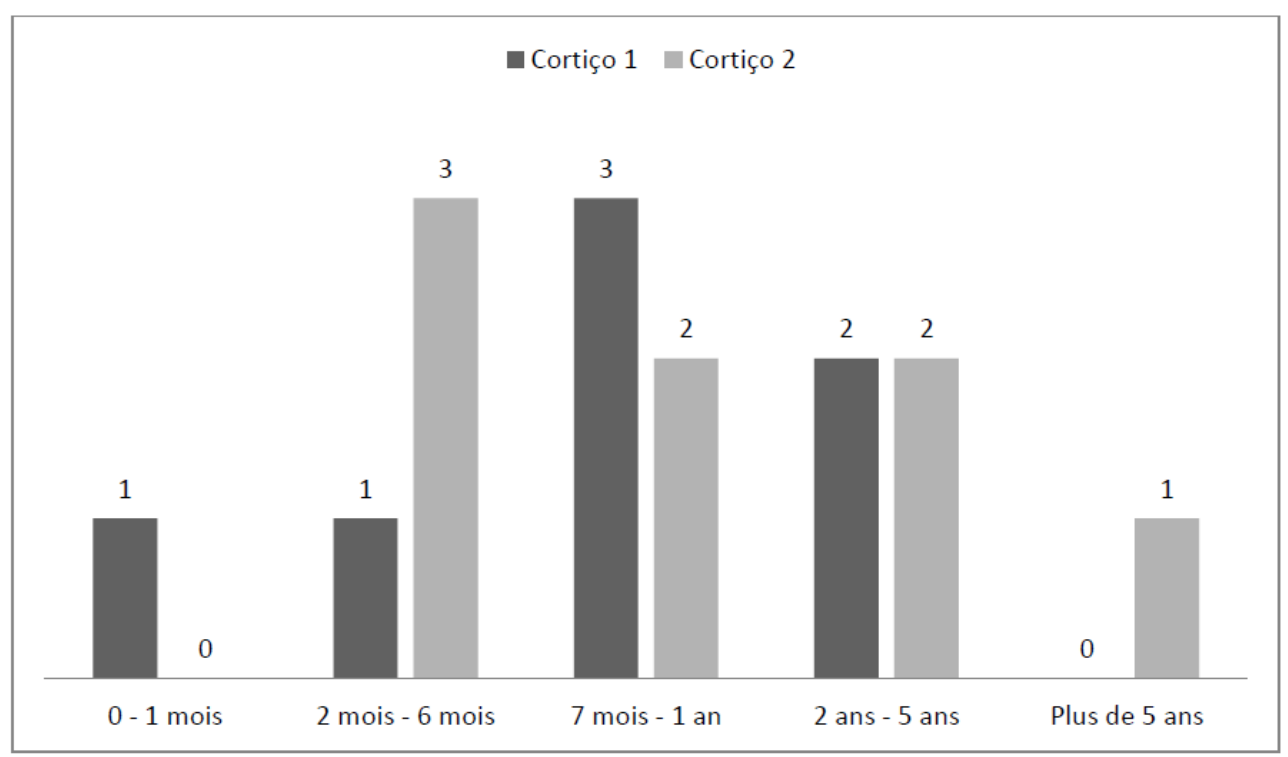

Réalisation : Auteure, Boa Vista - Recife, Mai 2013

Dans un pays où l'accès à la propriété est le fondement de la Nation [

Figure 6 1997], le cortiço apparaît donc comme un type de logement singulier, puisqu'il s'appuie sur le principe locatif. Cependant, en faisant ce choix résidentiel, ses habitants se démarquent $^{19}$ : entre la possibilité de ne pas payer de loyer dans d'autres types d'habitats populaires et celle de s'acquitter d'une somme mensuelle, ils font ce second choix : vivre dans des conditions de logement non moins précaires mais centrales dans la ville, s'assurant ainsi une insertion dans la ville formelle. Ce choix résidentiel, qui est donc aussi souvent un

\footnotetext{
${ }^{17}$ Guf

${ }^{18}$ Trou

${ }^{19}$ Ce choix est aussi, bien évidemment, contraint par les lois et les prix du marché pour cette population à très bas revenus, vivant avec la précarité de l'emploi qui caractérise l'emploi informel.
} 
choix professionnel, explique un mouvement d'ancrage dans un espace qui semblait initialement investi pour du temporaire.

Une installation dans le temporaire ? De l'appropriation de l'espace domestique à l'ancrage urbain

Plusieurs signes indiquent que l'habitat en cortiço, tout temporaire qu'il soit pensé, tout collectif qu'il soit, donne lieu à des dynamiques d'appropriation. Nous formulons même I'hypothèse que l'étude de la micro appropriation au sein du cortiço peut participer à mieux comprendre le rapport à la ville de ses habitants [Staszak, Collignon, 2003]. C'est en étudiant l'espace de l'intime et du lieu de vie quotidien que représente le logement que l'appréciation du rapport au reste de la ville peut être développée. Les rapports à l'espace intra-cortiço peuvent être corrélés aux rapports à l'espace du quartier, de la région urbaine (centre, périphérie, deuxième couronne...), voire de l'ensemble des espaces de la ville d'étude. Comment les appropriations de l'espace interne du cortiço peuvent-elles expliquer l'appropriation plus large à l'échelle de la ville?

Figure 7 : Appropriation de l'espace dans le cortiço et ancienneté dans le logement

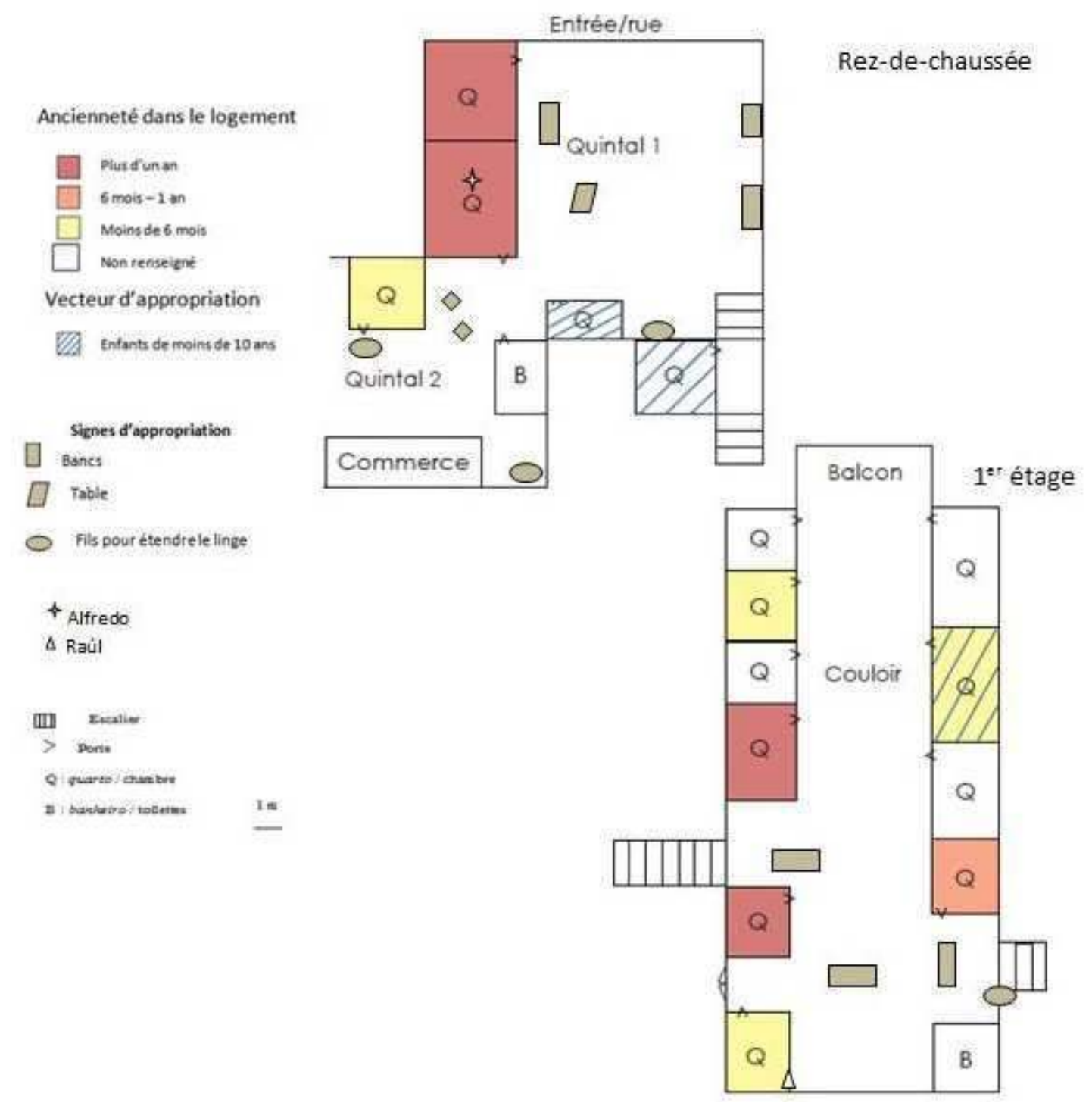


La Figure 7 croise l'ancienneté des habitants dans le cortiço avec certains signes identifiés comme des marqueurs d'appropriation de l'espace domestique, tels des bancs, des tables, des fils à linge... Bien qu'ils soient spécifiques à chaque cas d'étude, nous faisons l'hypothèse que ces indices démontrent une forme d'installation chez les populations résidentes en cortiço. Ainsi, tous les habitants résidents depuis plus de six mois dans ce cortiço de Recife ont installé soit des bancs, soit des tables, contrairement aux résidents plus récents. Cependant, un habitant de moins de six mois peut également avoir disposé des fils pour étendre le linge et déposé ses bicyclettes devant son quarto, ce qui marque aussi une appropriation précoce de leur logement. En outre, ce mobilier n'occupe pas l'espace privé du quarto mais investit l'espace collectif du couloir ou de la cour. De façon complexe, l'espace vécu - « l'espace tel qu'il est perçu et pratiqué par les êtres qui y vivent " [Staszak, 2003] - du cortiço se trouve donc être une imbrication d'espaces provisoires et prolongés, privés et collectifs, avec des délimitations plus ou moins fluctuantes, plus ou moins variables en fonction des modes d'appropriation qui s'appliquent et des temporalités résidentielles.

A une autre échelle, celle de la ville, d'autres signes indiquent un ancrage des populations du cortiço, associé paradoxalement à une forte mobilité résidentielle au sein du quartier. A la fois, la population est définie comme "fluctuante » [Suelly Gomes, Agent territorial District Sanitaire I- Boa Vista - Recife] ou " nomade ", et à la fois, elle présente de nombreux traits attestant d'un fort ancrage dans leur espace de vie, entendu comme "l'espace fréquenté et parcouru par chacun avec un minimum de régularité (...), [l']espace d'usage [composé] de lieux attractifs, de synapses, de nœuds autour desquels se cristallise l'existence individuelle : le logis, le cadre de travail, les espaces de loisir, de promenade, de rencontre» [Frémont, Chevalier, Hérin et Renard, 1984]. On peut alors préférer le terme de résidents en cortiço à celui d'habitants, étant entendu que l'on habite plusieurs parties de la ville mais que l'on ne réside qu'à un - voire deux - endroit [Lévy, 2003].

"Les personnes qui habitent ici sont, des itinérants, ceux qui vivent ici dans le Centre généralement sont des nomades, qui ont une rotativité importante. ${ }^{21}{ }^{\prime}$ [Entretien Carlos Antônio da Silva, Policier Civil, Boa Vista - Recife]

Cet ancrage est avant tout individuel : on ne peut pas parler d'inscription spatiale collective des résidents en cortiço, qui contribuerait par exemple à " l'existence d'une identité de groupe » et à un sentiment d'appartenance commun à l'espace [Fol, $2010: 4]$ étant donnée la "vulnérabilité identitaire " [Vidal, 2000] du sentiment de communauté dans l'habiter en cortiço. $^{22} \mathrm{Ni}$ les habitants des cortiços paulistes, ni ceux de Recife ne se sentent, ni se définissent comme tels; on ne constate ni autodénomination, ni reconnaissance d'appartenance à un groupe comme c'est le cas dans d'autres types d'habitats populaires telle la favela. Dans la société brésilienne, le terme utilisé en substitution à celui de favela négativement connoté est celui de comunidade [communauté] soulignant la mutualisation

\footnotetext{
${ }^{20} \mathrm{Si}$ les adresses précises apparaissent dans le travail de recherche initial, la publication incite à une certaine éthique envers le droit à l'anonymat.

21 “O pessoal que mora aqui é um [...] um nômade, quem mora aqui no Centro ele geralmente é nômade, e ele tem uma rotatividade importante."

${ }^{22}$ Cette vulnérabilité est à comprendre comme une faiblesse dans l'affirmation d'une identité qui se réfèrerait au type de logement; Vidal interroge lui cette vulnérabilité identitaire dans les communautés de type favelas dans le quartier de Brasilia Teimosa, à Recife.
} 
des voix et des actions en tant que demande d'un groupe aux intérêts communs et se reconnaissant comme tel. Dans certains cas, les habitants des cortiços disent habiter en pensão [pension], terme a priori moins connoté négativement mais qui ne révèle aucun sentiment d'appartenance à un groupe ni à une communauté. Au contraire, même, alors que le cortiço est un logement collectif où les espaces communs sont omniprésents, à commencer par l'espace intime des installations sanitaires, cette promiscuité quotidienne semble entraîner une forme d'individualisation des pratiques.

Comment l'appropriation du cortiço et l'ancrage personnel des habitants influencent-ils ensuite l'accès la ville et l'ancrage citadin des habitants?

\section{Le cortiço : sas pour l'accès à la ville?}

Tout comme le squat peut être un lieu d'émancipation [Bouillon, 2010 : 74], I'habiter en cortiço pourvoie des avantages en créant un levier vers un accès plus complet à la ville. L'étude du mode de fonctionnement et du rapport à la ville de ces populations habitantes tient de l'étude de formes invisibles, formes sociales (les populations) et formes physiques (le bâti même).

L'appropriation de l'espace du cortiço entraîne pour ses habitants un ancrage sous plusieurs formes, de l'ancrage résidentiel à l'ancrage citadin. A São Paulo, la ville et le quartier, inconnus à leur arrivée, sont accessibles aux nouveaux habitants via l'accès au logement, qui leur donne par la suite l'accès à l'emploi et aux services urbains. Certains connaissent particulièrement bien leur quartier et l'espace central de la ville, puisqu'ils les parcourent quotidiennement en exerçant leur métier de vendeurs ambulants. Dans ce contexte, le cortiço permet une installation rapide et directe dans le territoire urbain, sans passer par d'autres étapes, telles que le logement chez une connaissance ou encore en périphérie. Dans le cas pauliste, le cortiço joue donc un rôle de sas dans le processus d'ancrage résidentiel et d'ancrage citadin. Un logement situé en périphérie de la ville ne permettrait pas le même type d'ancrage citadin. Ainsi, paradoxalement, si l'idée d'ancrage semble liée à l'accès à la propriété dans d'autres types d'habitats populaires, dans le cas présent, le système locatif du cortiço n'est donc pas incompatible avec les processus d'ancrage sur le long terme. A Recife, la grande majorité de la population des cortiços étant originaire de Recife même, si ce n'est du quartier de Boa Vista [Erreur! Source du renvoi introuvable.], son ancrage citadin est à considérer à partir d'une certaine ancienneté dans la ville, la zone ou le quartier : la plupart des habitants interrogés fréquentent le quartier depuis plus de dix ans, démontrant ainsi leur ancrage dans la centralité de Recife. 
Figure 8 : Depuis quand vivez-vous dans le cortiço ? Ancienneté des résidents en cortiço dans le quartier de Boa Vista
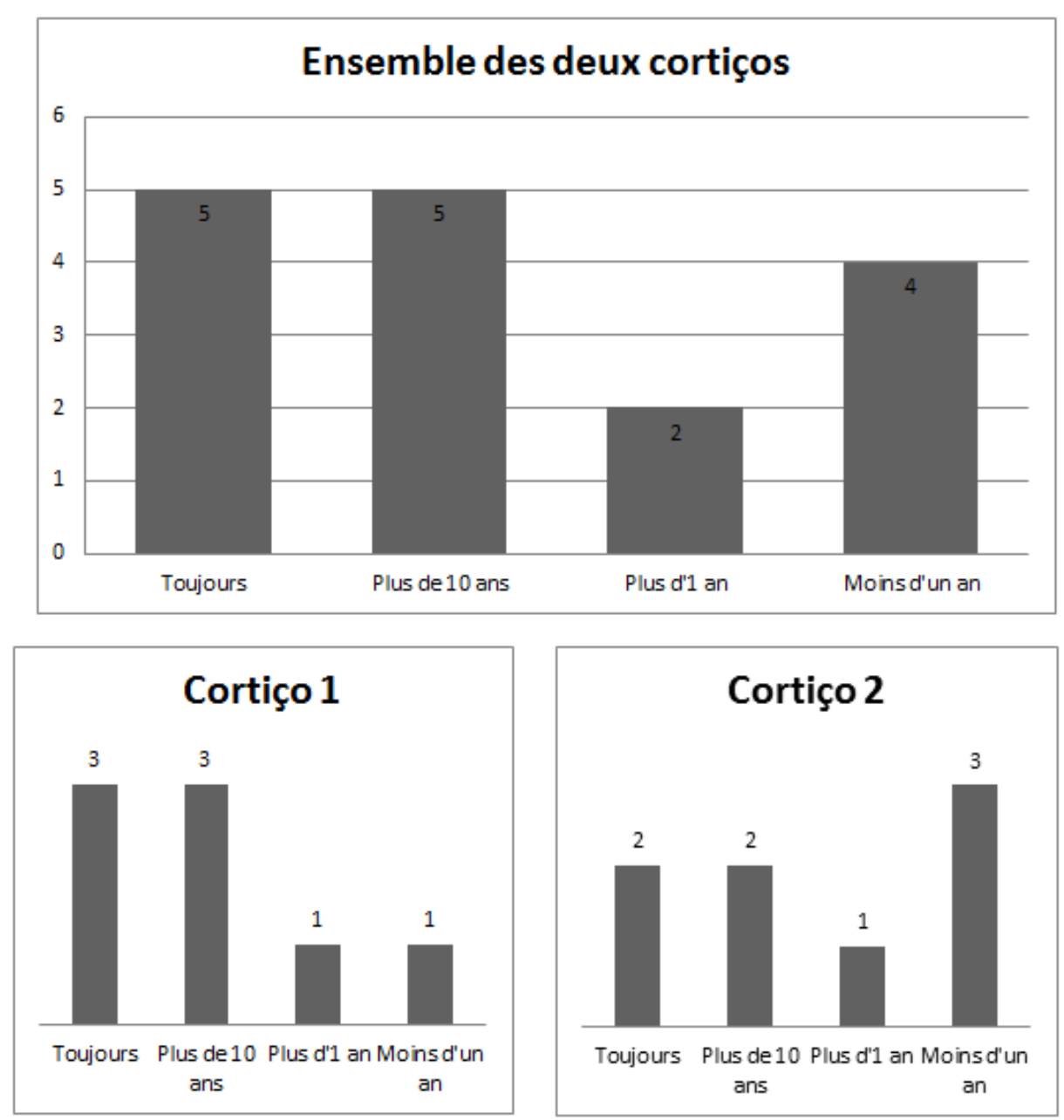

Réalisation : Auteure, Juillet 2013

Les habitants de cortiço, par ce choix de type de logement du centre, se dotent donc d'une ressource essentielle, celle de l'accès à la ville, permise notamment par la concentration de transports en commun. Cependant, leur espace de mobilité est davantage localisé dans les quartiers centraux que vers d'autres espaces plus lointains de la ville. En ce sens, il semble plus pertinent de convoquer ici la notion de motilité, définie par Kaufmann comme "la manière dont un individu ou un groupe fait sien le champ du possible en matière de mobilité et en fait usage pour développer des projets " [Kaufmann, 2004 : 6]. L'intérêt d'une telle définition réside pour nous dans son approche du potentiel, qui rappelle les stratégies résidentielles que développent les habitants en faveur de la centralité urbaine tout en étant très peu mobiles en dehors du quartier central, voire dans ce quartier central : « ce potentiel ne se transforme pas nécessairement en déplacements, il peut très bien être construit pour rester à court terme à l'état de potentiel afin de permettre, par exemple, une ouverture maximum à des opportunités futures " [ibid.]. Cette idée de potentiel est particulièrement présente dans les modes d'être en ville de la population en cortiço. La localisation centrale 
du logement et l'accès pédestre, proche et rapide aux aménités de la centralité urbaine qu'elle rend possible est un argument souvent évoqué par les habitants interrogés lors des questionnaires. Cependant, l'usage des espaces publics ou des lieux de l'espace sociétal à accès normé tels que les cafés, les centres commerciaux ou ceux à accès restreint comme les cinémas ou les théâtres n'est pas systématiquement effectif. Tout comme d'autres logements populaires, tel le squat [Bouillon, 2010], le cortiço " favorise [donc] l'insertion urbaine " pour se situer dans une centralité topographique. Mais la fréquentation des lieux associés à cette centralité reste parfois au stade de la potentialité d'usage. En cela, le cortiço apparaît comme un espace de l'entre-deux, où l'insertion dans la ville formelle n'induit pas systématiquement l'insertion dans les pratiques de cette ville formelle, comme si le regard était extérieur tout en étant à l'intérieur.

La motilité est à remettre dans le cadre plus large de la notion de capabilité [Sen, 1982], définie comme "la possibilité effective qu'un individu a de choisir entre diverses combinaisons de fonctionnements" ou encore "la possibilité pour les individus de faire des choix parmi les biens qu'ils jugent estimables et de les atteindre effectivement " [Sen, 1982]. Les habitants des cortiços sont physiquement proches de certains équipements urbains culturels, de récréation... - mais ils n'y ont pas accès, du fait de la censure et de l'autocensure. Toutefois, l'insertion dans la ville formelle prodigue aux habitants une adresse précise, référencée: cette relation au cadastre leur est fondamentale pour accéder à l'emploi, dont ne peuvent pas bénéficier les habitants d'une favela non intégrée officiellement, non reconnue dans le tissu urbain. Ce détail, qui n'en est pas un, donne aux habitants une visibilité sur le marché de l'emploi qui crédibilise leurs recherches. Alors que l'invisibilité peut desservir l'intérêt des habitants, au sens où elle ne permet pas une prise en compte du logement par les pouvoirs publics ou les ONG, elle permet d'éviter l'écueil de la stigmatisation ou de la marginalisation. L'habitat en cortiço résulte donc aussi d'un arbitrage individuel, qui renvoie plus largement à l'accès des habitants à la ville comme citadins. En brésilien, le terme cidadania fait converger en son sein ces deux notions de civilité et de citadinité - dans une acception plus large et qui rappelle les fondements du Droit à la Ville [Lefebvre, 1968]. Le potentiel de construction de la citadinité offert par le cortiço reste donc encore à analyser dans ce cas particulier brésilien.

\section{Eléments de conclusion}

Cet article, fondé sur deux expériences de terrain personnelles, révèle l'impact du contexte urbain dans lequel s'insère un même type de logement populaire, ici le cortiço, sur le mode de fonctionnement de ce dernier et les dynamiques qui lui sont associées dans l'être en ville. Deux cas de figures se sont distinguées dans l'étude réalisée auprès de ces deux réalités urbaines bien distinctes : celle de São Paulo, capitale économique du pays et celle de Recife, ville la plus importante de la région Nordeste considérée comme la plus pauvre. Dans le cas du cortiço pauliste, ce logement apparaît comme un entre-deux spatio-temporel pour les populations de néo-arrivants en provenance d'autres Etats de l'Union. C'est par le logement que se fait l'accès à la ville, au travail, aux différents services urbains... Dans le cas du cortiço recifense, ce logement n'a pas ce rôle "formateur » pour une population qui aurait à acquérir une connaissance de la citadinité, puisque il ne s'agit majoritairement pas de populations migrantes mais de personnes originaires de la ville de Recife et parfois du 
quartier même. A São Paulo, le cortiço paraît bien davantage jouer un rôle de sas spatiotemporel pour les habitants en provenance de tout le pays vers un accès à la ville.

L'hétérogénéité et la diversité de la population vivant en cortiço sont à souligner en termes de répartition sexuée, en termes d'écarts de tranches d'âges représentées, en termes de statut d'occupation et d'emploi... Ainsi, le cortiço comme lieu de l'entre-deux remet en question la catégorisation du type de population vivant dans ce type de logement populaire.

De plus, le cortiço apparait comme un espace de l'entre-deux sur le plan politicoadministratif, passant entre les mailles des lignes d'action des politiques publiques notamment, du fait de son insertion dans la ville formelle, du caractère ponctuel de sa localisation et de son régime de visibilité particulièrement effacé et discret dans le paysage urbain. Cette idée de l'entre-deux se retrouve également dans le fonctionnement des différentes échelles politico-administratives brésiliennes, mettant en évidence une extrême fragmentation des pouvoirs qui handicape fortement la coordination des actions aux différentes échelles [Fauré, 2004 : 13]. S'il est un espace de l'entre-deux politicoadministratif et invisible par bien des aspects, le cortiço reste toutefois une solution de logement populaire recherchée précisément car ce caractère de l'entre-deux et ce régime de visibilité particulier lui donnent une valeur ajoutée face à d'autres types de logement populaire. L'espace du cortiço est enfin un espace de la motilité, de la capabilité et des potentialités face à la ville dans laquelle il s'insère. Cette dimension vient interroger le degré de citadinité que les habitants peuvent acquérir d'un type de logement populaire à un autre. Le cortiço est donc un espace constitué de sas en son sein - entre espaces privatifs et espaces collectifs -, se révélant comme un sas à l'échelle des trajectoires de vie dans certains cas et comme un sas en termes d'accès à la ville et à toutes les aménités qui lui sont liées. Le cortiço se présente comme un double sas : un sas temporel, comme une étape provisoire, et un sas spatial qui positionne ses habitants dans un espace central a priori favorable à un meilleur accès à la ville en termes d'accessibilité à l'emploi, aux infrastructures et aux services urbains, et à la citadinité. L'étude gagnerait à être développée sur une temporalité plus étendue afin d'analyser les ouvertures prodiguées par ce sas que représente le cortiço pour ses habitants. Permet-il l'accès à d'autres types de logement : estil un sas vers d'autres types de logements populaires uniquement ou permet-il d'accéder à un logement qui ne soit pas de cette catégorie ? Nous évoquions la discrétion si ce n'est l'inexistence de l'action des politiques publiques face à la réalité des cortiços et, en même temps, l'absence d'action collective de la part des habitants pour revendiquer une position face aux autorités publiques : comment, dès lors, se construit la citadinité dans l'invisibilité, l'individualité et l'informalité ? si les pouvoirs publics venaient à intervenir et à investir de façon plus importante, cet espace présenterait-il toujours autant d'avantages ? Autant de points restant au stade de questionnements, qui permettraient de préciser les processus de transition et de médiation, qui se jouent dans l'entre-deux.

\section{Bibliographie}

AGIER M. (2008) Gérer les indésirables. Des camps de réfugiés au gouvernement humanitaire, Paris, Flammarion, "Bibliothèque des savoirs"

BERQUE A. (1995) Les raisons du paysage, de la Chine antique aux environnements de synthèse, Editions Hazan

BOUILLON F. (2011), Le squat : problème social ou lieu d'émancipation, Paris, Rue D'Ulm, 
collection "La rue ? Parlons-en!"

BURGESS EW., (2005) "La croissance de la ville; Introduction à un projet de recherche ", dans Grafmeyer Y., \& Joseph I., (dir). L'École de Chicago. Naissance de l'écologie urbaine, Aubier, Paris, 2005.

CAVALCANTI H., et alii (2008) Mosaico urbano do Recife. Inclusão/Exclusão socioambiental. Fundação Joaquim Nabuco. Ed. Massangana

CHABROL M., (2011) De nouvelles formes de gentrification ? Dynamiques résidentielles et commerciales dans le quartier de Château-Rouge (Paris), Thèse, Université de Poitiers

DI MÉO G., (2003). Géographie sociale et territoires. Paris, Nathan, Coll. Fac

FOL S., (2010) "Mobilité et ancrage dans les quartiers pauvres : les ressources de la proximité", Regards Sociologiques, $n^{\circ} 40,2010$, pp. 27-43

FREMONT A., CHEVALIER, J., HERIN, R., et RENARD, J. (1984) Géographie sociale. Paris, Masson

GERVAIS-LAMBONY P., (2007) Vies Citadines, Belin, Collection Mappemonde

KAUFMAN V., JEMELIN C., (2004) "La motilité, une forme de capital permettant d'éviter les irréversibilités socio-spatiales ?”, ESO - Le Mans - téléchargé le 10 Juin 2013, URL : http://eso.univ-lemans.fr/IMG/pdf/kv.pdf

LAWRENCE RJ,. (1982) “L'espace domestique : typologie et vécu, Cahiers internationaux de sociologie", vol. LXXII, Seuil

LEVY J., LUSSAULT M. [dir.], (2003) Dictionnaire de la géographie, de l'espace et des sociétés. Paris, Belin

LEVY JP., DUREAU F., [dir], (2002) L'accès à la ville : les mobilités spatiales en questions. L'Harmattan, Coll. Habitat et Sociétés

LEVY-VROELANT C., (2008) "Quartiers mixtes d'autrefois : la fonction sociale du logement ", Trajectoires familiales et espaces de vie en milieu urbain, Huitièmes entretiens Jacques Cartier, Yves Grafmeyer et Francine Dansereau éds, Presses Universitaires de Lyon.

MADORE F., PIHAN J., (2003) " Habitat et stratégies résidentielles"

LOPEZ, FRANÇA \& PRADO COSTA et alii, (2010) "Os Cortiços : A experência de São Paulo. Prefeitura de São Paulo." Téléchargé le 6 Janvier 2012,

URL : http://www.habisp.inf.br/theke/documentos/publicacoes/corticos/index.html, 2010.

MARICATO E., (1997) Habitação e cidade. São Paulo. Atual.

MICHEL X., (2007) "Paysage urbain : prémisses d'un renouvellement dans la géographie française, 1960-1980", Strates [En ligne], 13 | 2007, mis en ligne le 12 novembre 2008, Consulté le 13 août 2013. URL : http://strates.revues.org/5403

MONBEIG P., (1953) "La croissance de la ville de São Paulo ". Revue de Géographie Alpine, 41(1), 59-97.

PETCOU C, NICOLAS-LE STRAT P, PETRESCU D, MATTHYS K, DECK F, (2005) Interstices urbains temporaires, espaces interculturels en chantier, Lieux de proximité, Programme interdisciplinaire de recherche Art, architecture et paysage, RDS et ISCRA

RIMBERT S., (1973) “Approches des paysages ", L'Espace géographique, $\mathrm{n}^{\circ} 3$

SEN A., (1982) Poverty and Famines : An Essay on Entitlement and Deprivation, Oxford University Press

SIEVERTS T., (2004) Entre ville. Une lecture de la Zwischenstadt, Marseille, Editions Parenthèses

THERY H. (2007) " Chaves para a leitura do território paulista », Confins [En ligne], 1 | 2007.

VASSET P. (2007) Un livre blanc. Récit avec cartes, Paris, Fayard 
VAZ L.F. (1994). "Dos cortiços às favelas e aos edíficios de apartamentos - a modernização da moradia no Rio de Janeiro". Análise social. Vol. XXIX (3) 581-597

VIDAL D., (2000) La politique au quartier : Rapport sociaux et citoyenneté à Recife. Paris, Editions de la Maison des Sciences de l'Homme (Brasilia 14)

VOIROL Olivier, "Présentation " Visibilité et invisibilité : une introduction, Réseaux, 2005/1 $n^{\circ}$ 129-130, p. 9-36., consulté le 17 Décembre 2013 http://www.cairn.info/revue-reseaux2005-1-page-9.htm 